Supporting Information for

\title{
A Convenient Method for the Synthesis of 2,5-Difunctionalized Phospholes Bearing Ester Groups
}

Yoshihiro Matano,* Tooru Miyajima, Takashi Nakabuchi, Yuichiro Matsutani, and Hiroshi Imahori

Department of Molecular Engineering, Graduate School of Engineering, Kyoto University, Nishikyo-ku, Kyoto 615-8510, Japan

matano@scl.kyoto-u.ac.jp

Contents:

General:

SI-2

Synthesis and Characterization of 1, 4b-g, 6-10: SI-2

${ }^{1} \mathrm{H}$ and ${ }^{13} \mathrm{C}$ NMR charts of $\mathbf{4 b}, \mathbf{4 e - g , 7 - 1 0}$ 


\section{General}

All melting points are uncorrected. ${ }^{1} \mathrm{H},{ }^{13} \mathrm{C}\left\{{ }^{1} \mathrm{H}\right\}$, and ${ }^{31} \mathrm{P}\left\{{ }^{1} \mathrm{H}\right\}$ NMR spectra were recorded using $\mathrm{CDCl}_{3}$ as the solvent unless otherwise noted. Chemical shifts are reported as the relative value vs. tetramethylsilane $\left({ }^{1} \mathrm{H}\right.$ and $\left.{ }^{13} \mathrm{C}\right)$ and phosphonic acid $\left({ }^{31} \mathrm{P}\right)$. The ${ }^{1} \mathrm{H}$ and ${ }^{13} \mathrm{C}$ NMR charts of the key compounds $(\mathbf{4 b}, \mathbf{4 e}, \mathbf{4 f}, \mathbf{4 g}, \mathbf{7 - 1 0})$ are provided in Figures S1-S15. MALDI-TOF mass spectra were measured using CHCA as a matrix. IR spectra were observed as $\mathrm{KBr}$ pellets. All solvents were distilled from sodium benzophenone ketyl (ether, THF), sodium (hexane), or calcium hydride $\left(\mathrm{CH}_{2} \mathrm{Cl}_{2}\right.$, toluene) before use. All the reactions were performed under an argon or nitrogen atmosphere. Column chromatography was performed on silica gel or on a fast flow liquid chromatography system fitted with a silica gel column. Diynes 1a-h were prepared starting from 1,7-octadiyne (for 1a), 1,6-heptadiyne (for $\mathbf{1 b}, \mathbf{c}, \mathbf{e}-\mathbf{h}$ ), and dipropargyl ether (for 1d) according to Scheme S1. Other chemicals were of reagent grade quality, purchased commercially and used without further purification unless otherwise noted.

\section{Synthesis and Characterization of 1, 4b-g, 6-10}

Diethyl deca-2,8-diynedioate $\left(\mathbf{1 a}^{1}\right):{ }^{1} \mathrm{H}$ NMR $\delta 1.31(\mathrm{t}, 6 \mathrm{H}, J=7.2 \mathrm{~Hz}), 1.72(\mathrm{~m}, 4 \mathrm{H}), 2.39$ (m, 4H), $4.22(\mathrm{q}, 4 \mathrm{H}, J=7.2 \mathrm{~Hz})$.

Diethyl nona-2,7-diynedioate $\left(\mathbf{1 b}^{1}\right)$ : ${ }^{1} \mathrm{H}$ NMR $\delta 1.31(\mathrm{t}, 6 \mathrm{H}, J=7.1 \mathrm{~Hz}$ ), 1.86 (quin, $2 \mathrm{H}, J$ $=7.1 \mathrm{~Hz}), 2.49(\mathrm{t}, 4 \mathrm{H}, J=7.1 \mathrm{~Hz}), 4.22(\mathrm{q}, 4 \mathrm{H}, J=7.1 \mathrm{~Hz})$.

Dimethyl nona-2,7-diynedioate (1c ${ }^{2}$ ): ${ }^{1} \mathrm{H}$ NMR $\delta 1.86$ (quin, $2 \mathrm{H}, J=7.0 \mathrm{~Hz}$ ), 2.50 (t, 4H,

1 Jones, G. B.; Wright, J. M.; Plourde, G. W. II; Hynd,G.; Huber, R. S.; Mathews, J. E. J. Am. Chem. Soc. 2000, 122, 1937.

${ }^{2}$ Scheffer, J. R.; Wostradowski, R. A. J. Org. Chem. 1972, 37, 4317. 
$J=7.0 \mathrm{~Hz}), 3.77(\mathrm{~s}, 4 \mathrm{H})$.

Bis[3-(ethoxycarbonyl)propargyl] ether $\left(\mathbf{1 d}^{3}\right):{ }^{1} \mathrm{H}$ NMR $\delta 1.32(\mathrm{t}, 6 \mathrm{H}, J=7.2 \mathrm{~Hz}), 4.25$ $(\mathrm{q}, 4 \mathrm{H}, J=7.2 \mathrm{~Hz}), 4.41(\mathrm{~s}, 4 \mathrm{H})$.

1-Ethoxycarbonyl-7-phenyl-1,6-heptadiyne (1e): ${ }^{1} \mathrm{H}$ NMR $\delta 1.31(\mathrm{t}, 3 \mathrm{H}, J=7.1 \mathrm{~Hz})$, $1.89(\mathrm{tt}, 2 \mathrm{H}, J=7.1,7.1 \mathrm{~Hz}), 2.54(\mathrm{t}, 2 \mathrm{H}, J=7.1 \mathrm{~Hz}), 2.56(\mathrm{t}, 2 \mathrm{H}, J=7.1 \mathrm{~Hz}), 4.22(\mathrm{q}, 4 \mathrm{H}$, $J=7.1 \mathrm{~Hz}), 7.28(\mathrm{~m}, 3 \mathrm{H}, \mathrm{m}), 7.39(\mathrm{~m}, 2 \mathrm{H}) ;{ }^{13} \mathrm{C}\left\{{ }^{1} \mathrm{H}\right\} \mathrm{NMR} \delta 14.0,17.8,18.6,26.7,61.9$, 73.7, 81.7, 88.2, 88.3, 123.6, 127.8, 128.2, 131.6, 153.7; IR (KBr) $v_{\max } 1712(\mathrm{C}=\mathrm{O}) \mathrm{cm}^{-1}$; MS (MALDI-TOF) m/z $241\left(\mathrm{M}^{+}\right)$. Anal. Calcd for $\mathrm{C}_{16} \mathrm{H}_{16} \mathrm{O}_{2}$ : C, 79.97; H, 6.71. Found: C, 79.72; H, 6.68.

1-Ethoxycarbonyl-7-(2-pyridyl)-1,6-heptadiyne (1f): ${ }^{1} \mathrm{H}$ NMR $\delta 1.31$ (t, 3H, $J=7.2 \mathrm{~Hz}$ ), $1.92(\mathrm{tt}, 2 \mathrm{H}, J=7.0,7.0 \mathrm{~Hz}), 2.55(\mathrm{t}, 2 \mathrm{H}, J=7.0 \mathrm{~Hz}), 2.60(\mathrm{t}, 2 \mathrm{H}, J=7.0 \mathrm{~Hz}), 4.22(\mathrm{q}, 4 \mathrm{H}$, $J=7.2 \mathrm{~Hz}), 7.20(\mathrm{ddd}, 1 \mathrm{H}, J=7.6,4.9,1.6 \mathrm{~Hz}), 7.38(\mathrm{dd}, 1 \mathrm{H}, J=7.6,1.6 \mathrm{~Hz}), 7.63$ (ddd, $1 \mathrm{H}, J=7.6,7.6,1.8 \mathrm{~Hz}), 8.55(\mathrm{dd}, 1 \mathrm{H}, J=4.9,1.8 \mathrm{~Hz}) ;{ }^{13} \mathrm{C}\left\{{ }^{1} \mathrm{H}\right\} \mathrm{NMR} \delta 14.1,18.0,18.6$, $26.4,61.9,73.8,81.4,87.9,88.8,122.4,126.8,136.0,143.4,149.8,153.6$; IR (KBr) $v_{\max }$ $1709(\mathrm{C}=\mathrm{O}) \mathrm{cm}^{-1}$; MS (MALDI-TOF) $\mathrm{m} / \mathrm{z} 242\left(\mathrm{M}^{+}\right)$. Anal. Calcd for $\mathrm{C}_{15} \mathrm{H}_{15} \mathrm{NO}_{2}$ : C, 74.67; H, 6.27; N, 5.81. Found: C, 74.68; H, 6.22; N,5.63.

1-Ethoxycarbonyl-7-(2-thienyl)-1,6-heptadiyne (1g): ${ }^{1} \mathrm{H}$ NMR $\delta 1.31$ (t, 3H, $J=7.1 \mathrm{~Hz}$ ), $1.88(\mathrm{tt}, 2 \mathrm{H}, J=7.1,7.1 \mathrm{~Hz}), 2.52(\mathrm{t}, 2 \mathrm{H}, J=7.1 \mathrm{~Hz}), 2.55(\mathrm{t}, 2 \mathrm{H}, J=7.1 \mathrm{~Hz}), 4.22(\mathrm{q}, 4 \mathrm{H}$, $J=7.1 \mathrm{~Hz}), 6.94(\mathrm{dd}, 1 \mathrm{H}, J=5.2,3.7 \mathrm{~Hz}), 7.13(\mathrm{dd}, 1 \mathrm{H}, J=3.7,1.1 \mathrm{~Hz}), 7.19(\mathrm{dd}, 1 \mathrm{H}, J=$ 5.2, 1.1 Hz); ${ }^{13} \mathrm{C}\left\{{ }^{1} \mathrm{H}\right\}$ NMR $\delta 14.2,17.9,18.9,26.6,61.8,73.7,74.8,87.9,92.2,123.5$, 126.1, 126.7, 131.2, 153.5; IR (KBr) $v_{\max } 1710(\mathrm{C}=\mathrm{O}) \mathrm{cm}^{-1}$; MS (MALDI-TOF) $\mathrm{m} / \mathrm{z} 246$ $\left(\mathrm{M}^{+}\right)$. Anal. Calcd for $\mathrm{C}_{14} \mathrm{H}_{14} \mathrm{O}_{2} \mathrm{~S}: \mathrm{C}, 68.26 ; \mathrm{H}$, 5.73. Found: $\mathrm{C}, 68.23 ; \mathrm{H}, 5.52$.

1-(9-Anthryl)-7-ethoxycarbonyl-1,6-heptadiyne (1h): $\mathrm{Mp} 42-43{ }^{\circ} \mathrm{C} ;{ }^{1} \mathrm{H} \mathrm{NMR} \delta 1.31$ (t,

${ }^{3}$ Yamamoto, Y.; Nagata, A.; Nagata, H.; Ando, Y.; Arikawa, Y.; Tatsumi, K.; Itoh, K. Chem. Eur. J. 2003, 9, 2469. 
$3 \mathrm{H}, J=7.1 \mathrm{~Hz}$ ), 2.10 (tt, $2 \mathrm{H}, J=7.0,7.0 \mathrm{~Hz}$ ), 2.70 and 2.91 (each t, $2 \mathrm{H}, J=7.0 \mathrm{~Hz}$ ), 4.23 (q, 4H, $J=7.1 \mathrm{~Hz}$ ), 7.46-7.58 (m, 4H), 7.99 (d, 2H, $J=8.4 \mathrm{~Hz}$ ), 8.39 (s, 1H), 8.51 (d, 2H, $J$ $=8.4 \mathrm{~Hz}) ;{ }^{13} \mathrm{C}\left\{{ }^{1} \mathrm{H}\right\}$ NMR $\delta 14.0,18.1,19.4,27.0,61.8,73.8,78.2,87.9,99.6,117.6,125.4$, $126.2,126.5,126.9,128.5,131.0,132.5,153.5$; IR (neat) $v_{\max } 1708(\mathrm{C}=\mathrm{O}) \mathrm{cm}^{-1}$; MS (MALDI-TOF) $\mathrm{m} / \mathrm{z} 340\left(\mathrm{M}^{+}\right)$. Although spectroscopic data clearly supported a high state of purity of $\mathbf{1 h}$, we were not successful in obtaining satisfactory analytical data of an accuracy within $\pm 0.4 \%$.
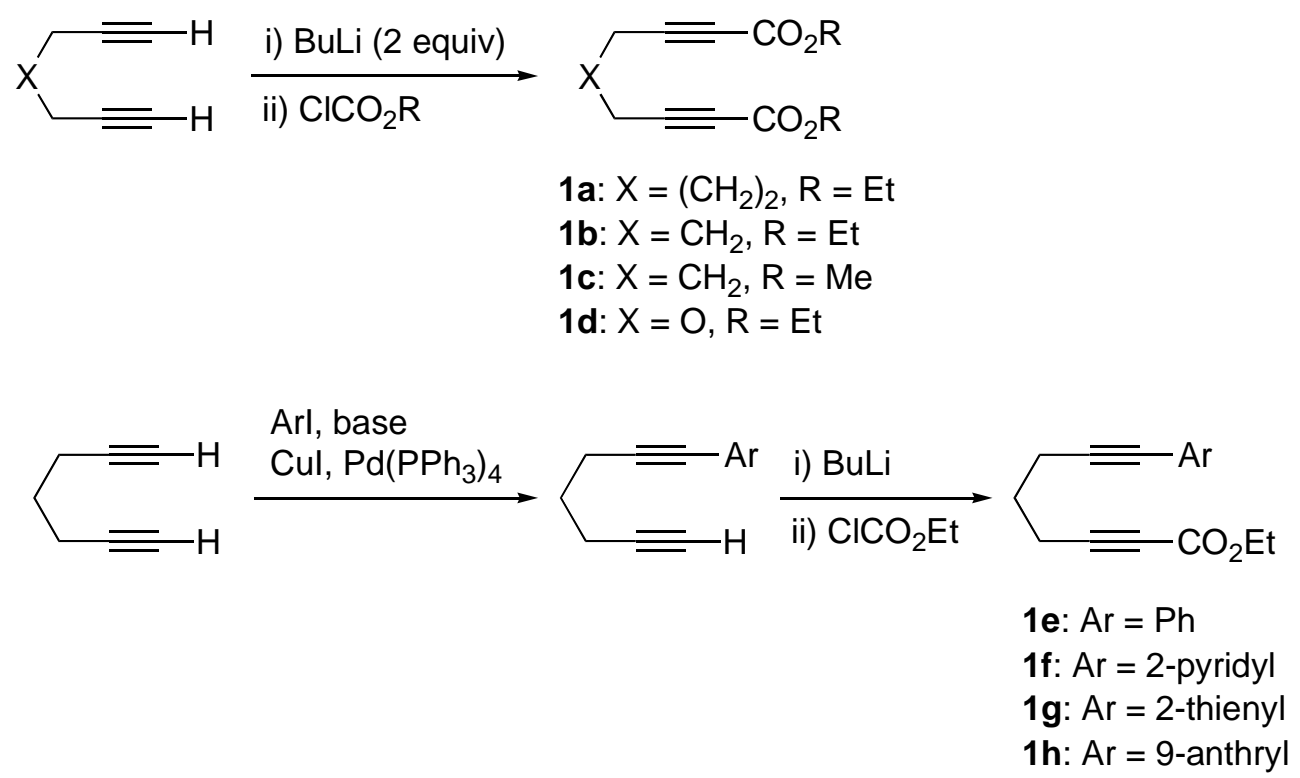

SCHEME S1. Synthesis of 1a-h.

Phosphole 4b: Mp 75-76 ${ }^{\circ} \mathrm{C} ;{ }^{1} \mathrm{H}$ NMR $\delta 1.17$ (t, 6H, ${ }^{1} \mathrm{~J}=6.4 \mathrm{~Hz}$ ), 2.33 (br, 2H), 2,80 (br, 2H), 3.13 (br, 2H), 4.15 (br, 4H), 7.27-7.42 (m, 5H); ${ }^{13} \mathrm{C}\left\{{ }^{1} \mathrm{H}\right\}$ NMR $\delta 14.1,28.3,30.1,60.5$, $128.4\left(\mathrm{~d},{ }^{3} J_{\mathrm{P}-\mathrm{C}}=8.7 \mathrm{~Hz}\right), 129.8\left(\mathrm{~d},{ }^{4} J_{\mathrm{P}-\mathrm{C}}=1.9 \mathrm{~Hz}\right), 132.9,133.7\left(\mathrm{~d},{ }^{2} J_{\mathrm{P}-\mathrm{C}}=19.9 \mathrm{~Hz}\right), 164.7$ $\left(\mathrm{d},{ }^{2} J_{\mathrm{P}-\mathrm{C}}=20.4 \mathrm{~Hz}\right), 167.3\left(\mathrm{~d},{ }^{2} J_{\mathrm{P}-\mathrm{C}}=10.6 \mathrm{~Hz}\right) ;{ }^{31} \mathrm{P}\left\{{ }^{1} \mathrm{H}\right\} \mathrm{NMR} \delta+32.7 ; \mathrm{IR}(\mathrm{KBr}) v_{\max } 1703$, $1689(\mathrm{C}=\mathrm{O}) \mathrm{cm}^{-1}$; MS (MALDI-TOF) $\mathrm{m} / \mathrm{z} 345\left([\mathrm{M}+\mathrm{H}]^{+}\right)$. Anal. Calcd for $\mathrm{C}_{11} \mathrm{H}_{21} \mathrm{O}_{4} \mathrm{P}: \mathrm{C}$, 66.27 ; H, 6.15; P, 9.00. Found: C, 66.13; H, 6.36; P, 8.75. The ipso carbon of the phenyl 
group could not be detected clearly in the ${ }^{13} \mathrm{C}$ NMR.

Phosphole 4c : Mp 107-108 ${ }^{\circ} \mathrm{C} ;{ }^{1} \mathrm{H}$ NMR $\delta 2.33$ (br, 2H), 2,81 (br, 2H), 3.13 (br, 2H), 3.69 (s, 6H), 7.28-7.40 (m, 5H); ${ }^{13} \mathrm{C}\left\{{ }^{1} \mathrm{H}\right\}$ NMR $\delta 28.3,30.1,51.7,128.6\left(\mathrm{~d},{ }^{3} \mathrm{~J}_{\mathrm{P}-\mathrm{C}}=8.7 \mathrm{~Hz}\right)$, 129.8, $129.9\left(\mathrm{~d},{ }^{1} J_{\mathrm{P}-\mathrm{C}}=8.7 \mathrm{~Hz}\right), 132.5\left(\mathrm{~d},{ }^{1} J_{\mathrm{P}-\mathrm{C}}=3.1 \mathrm{~Hz}\right), 133.6\left(\mathrm{~d},{ }^{2} J_{\mathrm{P}-\mathrm{C}}=19.9 \mathrm{~Hz}\right), 165.1$ $\left(\mathrm{d},{ }^{2} J_{\mathrm{P}-\mathrm{C}}=20.5 \mathrm{~Hz}\right), 167.6\left(\mathrm{~d},{ }^{2} J_{\mathrm{P}-\mathrm{C}}=9.9 \mathrm{~Hz}\right) ;{ }^{31} \mathrm{P}\left\{{ }^{1} \mathrm{H}\right\} \mathrm{NMR} \delta+32.6$; IR $(\mathrm{KBr}) v_{\max } 1699$ $(\mathrm{C}=\mathrm{O}) \mathrm{cm}^{-1}$; MS (MALDI-TOF) $\mathrm{m} / \mathrm{z} 317\left(\mathrm{M}^{+}\right)$. Anal. Calcd for $\mathrm{C}_{9} \mathrm{H}_{17} \mathrm{O}_{4} \mathrm{P}: \mathrm{C}, 64.56 ; \mathrm{H}$, 5.42; P, 9.79. Found: C, 64.44; H, 5.57; P, 9.65 .

Phosphole 4d: Mp 89-90 ${ }^{\circ} \mathrm{C} ;{ }^{1} \mathrm{H}$ NMR $\delta 1.19$ (t, 6H, $J=7.1 \mathrm{~Hz}$ ), 4.17 (q, 4H, $J=7.1 \mathrm{~Hz}$ ), $4.97(\mathrm{~m}, 4 \mathrm{H}), 7.31-7.45(\mathrm{~m}, 5 \mathrm{H}) ;{ }^{13} \mathrm{C}\left\{{ }^{1} \mathrm{H}\right\} \mathrm{NMR} \delta 14.1,61.0,69.6,128.7\left(\mathrm{~d},{ }^{3} J_{\mathrm{P}-\mathrm{C}}=9.3\right.$ $\mathrm{Hz}), 130.3,132.1,133.9\left(\mathrm{~d},{ }^{2} J_{\mathrm{P}-\mathrm{C}}=20.5 \mathrm{~Hz}\right), 161.6\left(\mathrm{~d},{ }^{1} J_{\mathrm{P}-\mathrm{C}}=10.6 \mathrm{~Hz}\right), 163.8\left(\mathrm{~d},{ }^{2} J_{\mathrm{P}-\mathrm{C}}=\right.$ $15.6 \mathrm{~Hz}) ;{ }^{31} \mathrm{P}\left\{{ }^{1} \mathrm{H}\right\} \mathrm{NMR} \delta+40.5$; IR $(\mathrm{KBr}) v_{\max } 1690(\mathrm{C}=\mathrm{O}) \mathrm{cm}^{-1}$; MS (MALDI-TOF) $\mathrm{m} / \mathrm{z}$ $347\left([\mathrm{M}+\mathrm{H}]^{+}\right)$. Anal. Calcd for $\mathrm{C}_{10} \mathrm{H}_{19} \mathrm{O}_{5} \mathrm{P}: \mathrm{C}, 62.43 ; \mathrm{H}, 5.53 ; \mathrm{P}, 8.94$. Found: $\mathrm{C}, 62.16 ; \mathrm{H}$, 5.73; P, 8.66. The ipso carbon of the phenyl group could not be detected clearly in the ${ }^{13} \mathrm{C}$ NMR.

Phosphole 4e: Mp 135-136 ${ }^{\circ} \mathrm{C} ;{ }^{1} \mathrm{H}$ NMR $\delta 1.20$ (t, 3H, J = $7.3 \mathrm{~Hz}$ ), 2.35 (m, 2H), 2,653.15 (m, 4H), 4.14 (m, 2H), 7.17-7.24 (m, 4H), 7.27-7.31 (m, 2H), 7.39 (m, 2H), 7.49 (m, $2 \mathrm{H}) ;{ }^{13} \mathrm{C}\left\{{ }^{1} \mathrm{H}\right\} \mathrm{NMR} \delta 14.3,29.1,29.2,30.0,60.0,126.1,127.4,128.0\left(\mathrm{~d},{ }^{3} J_{\mathrm{P}-\mathrm{C}}=10.6 \mathrm{~Hz}\right)$, $128.5\left(\mathrm{~d},{ }^{3} J_{\mathrm{P}-\mathrm{C}}=8.8 \mathrm{~Hz}\right), 128.6,129.3,131.7\left(\mathrm{~d},{ }^{1} J_{\mathrm{P}-\mathrm{C}}=12.3 \mathrm{~Hz}\right), 133.6\left(\mathrm{~d},{ }^{2} J_{\mathrm{P}-\mathrm{C}}=19.3\right.$ $\mathrm{Hz}), 136.2\left(\mathrm{~d},{ }^{2} J_{\mathrm{P}-\mathrm{C}}=17.4 \mathrm{~Hz}\right), 144.6,153.1\left(\mathrm{~d},{ }^{2} J_{\mathrm{P}-\mathrm{C}}=8.1 \mathrm{~Hz}\right), 165.1\left(\mathrm{~d},{ }^{2} J_{\mathrm{P}-\mathrm{C}}=21.7 \mathrm{~Hz}\right)$, $170.1\left(\mathrm{~d},{ }^{2} J_{\mathrm{P}-\mathrm{C}}=11.8 \mathrm{~Hz}\right) ;{ }^{31} \mathrm{P}\left\{{ }^{1} \mathrm{H}\right\} \mathrm{NMR} \delta+32.0 ; \mathrm{IR}(\mathrm{KBr}) v_{\max } 1690(\mathrm{C}=\mathrm{O}) \mathrm{cm}^{-1} ; \mathrm{MS}$ (MALDI-TOF) $\mathrm{m} / \mathrm{z} 348\left(\mathrm{M}^{+}\right.$). Anal. Calcd for $\mathrm{C}_{22} \mathrm{H}_{21} \mathrm{O}_{2} \mathrm{P}: \mathrm{C}, 75.85 ; \mathrm{H}, 6.08$; $\mathrm{P}, 8.89$.

Found: C, 75.55; H, 6.25; P, 8.86 .

Phosphole 4f: Mp $135-136{ }^{\circ} \mathrm{C} ;{ }^{1} \mathrm{H}$ NMR $\delta 1.19$ (t, 3H, $J=7.2 \mathrm{~Hz}$ ), 2.37 (m, 2H), 2.75-3.25 (m, 4H), 4.15 (m, 2H), 7.06 (m, 1H), 7.19-7.28 (m, 3H), 7.41-7.46 (m, 2H), 7.47 (m, 1H), $7.56(\mathrm{~m}, 1 \mathrm{H}), 8.53(\mathrm{~m}, 1 \mathrm{H}) ;{ }^{13} \mathrm{C} \mathrm{NMR} \delta 14.3,28.9,29.8,30.0,60.1,121.4,122.1\left(\mathrm{~d},{ }^{3} J_{\mathrm{P}-\mathrm{C}}=\right.$ 
$7.2 \mathrm{~Hz}), 128.2\left(\mathrm{~d},{ }^{3} J_{\mathrm{P}-\mathrm{C}}=8.4 \mathrm{~Hz}\right), 129.2\left(\mathrm{~d},{ }^{4} \mathrm{~J}_{\mathrm{P}-\mathrm{C}}=1.7 \mathrm{~Hz}\right), 131.8\left(\mathrm{~d},{ }^{1} J_{\mathrm{P}-\mathrm{C}}=12.8 \mathrm{~Hz}\right)$, $133.6\left(\mathrm{~d},{ }^{2} J_{\mathrm{P}-\mathrm{C}}=19.5 \mathrm{~Hz}\right), 136.2\left(\mathrm{~d},{ }^{4} J_{\mathrm{P}-\mathrm{C}}=1.1 \mathrm{~Hz}\right), 143.7,149.6,154.5\left(\mathrm{~d},{ }^{2} J_{\mathrm{P}-\mathrm{C}}=17.8\right.$ $\mathrm{Hz}), 156.3\left(\mathrm{~d},{ }^{2} J_{\mathrm{P}-\mathrm{C}}=8.4 \mathrm{~Hz}\right), 165.0\left(\mathrm{~d},{ }^{2} J_{\mathrm{P}-\mathrm{C}}=21.2 \mathrm{~Hz}\right), 169.2\left(\mathrm{~d},{ }^{2} J_{\mathrm{P}-\mathrm{C}}=11.7 \mathrm{~Hz}\right) ;{ }^{31} \mathrm{P}$ NMR $\delta+32.6$; IR (KBr) $v_{\max } 1695(\mathrm{C}=\mathrm{O}) \mathrm{cm}^{-1}$; MS (MALDI-TOF) m/z $350\left(\mathrm{M}^{+}\right)$. Anal. Calcd for $\mathrm{C}_{21} \mathrm{H}_{20} \mathrm{NO}_{2} \mathrm{P}:$ C, 72.20; H, 5.77; N, 4.01; P, 8.89. Found: C, 72.10; H, 5.79; N, 3.97; P, 8.76. One of the $\alpha$ carbons could not be detected clearly in the ${ }^{13} \mathrm{C}$ NMR.

Phosphole 4g: Mp 131-132 ${ }^{\circ} \mathrm{C} ;{ }^{1} \mathrm{H}$ NMR $\delta 1.18$ (t, 3H, $\left.J=7.1 \mathrm{~Hz}\right), 2.39$ (m, 2H), 2.65-3.20 (m, 4H), $4.12(\mathrm{~m}, 2 \mathrm{H}), 6.93(\mathrm{~m}, 1 \mathrm{H}), 7.04(\mathrm{~m}, 1 \mathrm{H}), 7.27(\mathrm{~m}, 1 \mathrm{H}), 7.29(\mathrm{~m}, 3 \mathrm{H})$, $7.45(\mathrm{~m}, 2 \mathrm{H}) ;{ }^{13} \mathrm{C}\left\{{ }^{1} \mathrm{H}\right\}$ NMR $\delta 14.2,28.7,29.1,30.2,60.0,125.1,126.2\left(\mathrm{~d},{ }^{5} J_{\mathrm{P}-\mathrm{C}}=1.9 \mathrm{~Hz}\right)$, $126.4\left(\mathrm{~d},{ }^{3} J_{\mathrm{P}-\mathrm{C}}=8.0 \mathrm{~Hz}\right), 127.7,128.6\left(\mathrm{~d},{ }^{3} J_{\mathrm{P}-\mathrm{C}}=8.8 \mathrm{~Hz}\right), 129.7,132.0\left(\mathrm{~d},{ }^{1} J_{\mathrm{P}-\mathrm{C}}=13.7 \mathrm{~Hz}\right)$, $133.8\left(\mathrm{~d},{ }^{2} J_{\mathrm{P}-\mathrm{C}}=19.9 \mathrm{~Hz}\right), 137.9\left(\mathrm{~d},{ }^{1} J_{\mathrm{P}-\mathrm{C}}=3.7 \mathrm{~Hz}\right), 139.5\left(\mathrm{~d},{ }^{2} J_{\mathrm{P}-\mathrm{C}}=21.2 \mathrm{~Hz}\right), 152.1(\mathrm{~d}$, $\left.{ }^{2} J_{\mathrm{P}-\mathrm{C}}=8.1 \mathrm{~Hz}\right), 165.0\left(\mathrm{~d},{ }^{2} J_{\mathrm{P}-\mathrm{C}}=21.7 \mathrm{~Hz}\right), 170.3\left(\mathrm{~d},{ }^{2} J_{\mathrm{P}-\mathrm{C}}=10.6 \mathrm{~Hz}\right) ;{ }^{31} \mathrm{P}\left\{{ }^{1} \mathrm{H}\right\} \mathrm{NMR} \delta+$ 33.4; IR $v_{\max } 1690(\mathrm{C}=\mathrm{O}) \mathrm{cm}^{-1}$; MS (MALDI-TOF) $\mathrm{m} / \mathrm{z} 354\left(\mathrm{M}^{+}\right)$. Anal. Calcd for $\mathrm{C}_{22} \mathrm{H}_{19} \mathrm{O}_{2} \mathrm{PS}$ : C, 67.78; H, 5.40; P, 8.74. Found: C, 67.63; H, 5.46; P, 8.45.

Phospholes 6 and 7: To a solution of $\mathbf{4 b}(300 \mathrm{mg}, 0.87 \mathrm{mmol})$ in $10 \mathrm{~mL}$ of hexane was added a hexane solution of DIBAL $(1.0 \mathrm{M}, 3.5 \mathrm{~mL}, 3.5 \mathrm{mmol})$ at $-78^{\circ} \mathrm{C}$. After stirring for 1 $\mathrm{h}$ at $-50{ }^{\circ} \mathrm{C}$, elemental sulfur $(40 \mathrm{mg}, 1.3 \mathrm{mmol})$ was added, and the resulting mixture was allowed to warm slowly to room temperature. A saturated $\mathrm{NH}_{4} \mathrm{Cl}$ solution $(5 \mathrm{~mL})$ was then added, and the mixture was filtered through a Celite bed. The organic phase was separated from the filtrate, and the aqueous phase was extracted with $\mathrm{Et}_{2} \mathrm{O}(5 \mathrm{~mL} \times 3)$. The combined organic extracts were washed with brine $(20 \mathrm{~mL})$, dried over $\mathrm{Na}_{2} \mathrm{SO}_{4}$, and evaporated to give a crude product, which was recrystallized from EtOAc/hexane to give 7 (160 mg, 0.55 mmol, 63\%) as a pale yellow solid. When the reaction was quenched without adding elemental sulfur, the ${ }^{3}$-phosphine 6 was obtained as a rather air-sensitive substance. The 
structure of 6 was characterized on the basis of ${ }^{1} \mathrm{H}$ NMR and MS.

Phosphole 6: ${ }^{1} \mathrm{H}$ NMR $\delta 1.77$ (br, 2H), 2.14 (m, 1H), 2.28 (m, 1H), 2.45 (m, 2H), 2.64 (m, 2H), $4.38(\mathrm{~m}, 2 \mathrm{H}), 4.52(\mathrm{~m}, 2 \mathrm{H}), 7.28-7.40(\mathrm{~m}, 5 \mathrm{H})$; MS (MALDI-TOF) m/z 503 $\left([2 \mathrm{M}-\mathrm{OH}]^{+}\right)$.

Phosphole 7: Mp 131-132 ${ }^{\circ} \mathrm{C} ;{ }^{1} \mathrm{H}$ NMR $\delta 2.03$ (t, 2H, $\left.J=6.0 \mathrm{~Hz}\right), 2.10-2.27(\mathrm{~m}, 2 \mathrm{H})$, 2.58-2.73 (m, 4H), 4.38-4.52 (m, 4H), 7.43-7.55 (m, 3H), 7.82-7.88 (m, 2H); ${ }^{13} \mathrm{C}\left\{{ }^{1} \mathrm{H}\right\}$ NMR $\delta 27.0\left(\mathrm{~d},{ }^{4} J_{\mathrm{P}-\mathrm{C}}=1.8 \mathrm{~Hz}\right), 27.4\left(\mathrm{~d},{ }^{3} J_{\mathrm{P}-\mathrm{C}}=11.2 \mathrm{~Hz}\right), 57.6\left(\mathrm{~d},{ }^{2} J_{\mathrm{P}-\mathrm{C}}=15.6 \mathrm{~Hz}\right), 127.6$ $\left(\mathrm{d},{ }^{1} J_{\mathrm{P}-\mathrm{C}}=70.3 \mathrm{~Hz}\right), 128.9\left(\mathrm{~d},{ }^{2} J_{\mathrm{P}-\mathrm{C}}=12.4 \mathrm{~Hz}\right), 130.0,130.3\left(\mathrm{~d},{ }^{3} J_{\mathrm{P}-\mathrm{C}}=11.9 \mathrm{~Hz}\right), 132.2(\mathrm{~d}$, $\left.{ }^{4} J_{\mathrm{P}-\mathrm{C}}=2.4 \mathrm{~Hz}\right), 157.3\left(\mathrm{~d},{ }^{2} J_{\mathrm{P}-\mathrm{C}}=23.0 \mathrm{~Hz}\right) ;{ }^{31} \mathrm{P}\left\{{ }^{1} \mathrm{H}\right\} \mathrm{NMR} \delta+67.5 ; \mathrm{IR}(\mathrm{KBr}) v_{\max } 3390$ $(\mathrm{OH}) \mathrm{cm}^{-1}$; MS (MALDI-TOF) $\mathrm{m} / \mathrm{z} 292\left(\mathrm{M}^{+}\right)$. HRMS: Calcd for $\mathrm{C}_{15} \mathrm{H}_{18} \mathrm{O}_{2} \mathrm{PS}\left([\mathrm{M}+\mathrm{H}]^{+}\right)$, 293.0765; Found, 293.0766.

Phosphole 8: To a solution of $\mathbf{4 b}(1.17 \mathrm{~g}, 3.40 \mathrm{mmol})$ in $7 \mathrm{~mL}$ of THF was added $\mathrm{MeMgBr}$ $\left(15 \mathrm{~mL}, 0.96 \mathrm{M}\right.$ in THF, $14 \mathrm{mmol}$ ) at $-78^{\circ} \mathrm{C}$. The mixture was gradually warmed to room temperature during $30 \mathrm{~min}$, and stirred for an additional $30 \mathrm{~min}$ at $50^{\circ} \mathrm{C}$. The mixture was then acidified by sat. $\mathrm{NH}_{4} \mathrm{Cl}$ aq $(3 \mathrm{~mL})$ at $0{ }^{\circ} \mathrm{C}$, and elemental sulfur $(160 \mathrm{mg}, 5.0 \mathrm{mmol})$ was added at the same temperature. After stirring for $30 \mathrm{~min}$, an aqueous phase was extracted with ether. The organic extracts were combined, washed with brine, dried over $\mathrm{Na}_{2} \mathrm{SO}_{4}$, and evaporated to give an oily residue, which was subjected on silica gel column chromatography (hexane/EtOAc $\left.=2 / 1: R_{\mathrm{f}}=0.2\right)$ to afford 8 as a pale yellow solid (475 $\mathrm{mg}$, $1.36 \mathrm{mmol}, 40 \%$ ). Mp $133-134{ }^{\circ} \mathrm{C} ;{ }^{1} \mathrm{H}$ NMR $\oint 1.28(\mathrm{~s}, 6 \mathrm{H}), 1.43(\mathrm{~s}, 6 \mathrm{H}), 2.12-2.18(\mathrm{~m}, 2 \mathrm{H})$, 2.64-2.69 (m, 4H), $3.03(\mathrm{~s}, 2 \mathrm{H}), 7.26-7.49(\mathrm{~m}, 3 \mathrm{H}), 7.85-7.91(\mathrm{~m}, 2 \mathrm{H}) ;{ }^{13} \mathrm{C}$ NMR $\delta 27.1(\mathrm{~d}$, $\left.{ }^{4} J_{\mathrm{P}-\mathrm{C}}=1.7 \mathrm{~Hz}\right), 28.5\left(\mathrm{~d},{ }^{3} J_{\mathrm{P}-\mathrm{C}}=12.3 \mathrm{~Hz}\right), 29.8\left(\mathrm{~d},{ }^{3} J_{\mathrm{P}-\mathrm{C}}=1.6 \mathrm{~Hz}\right), 31.3\left(\mathrm{~d},{ }^{3} J_{\mathrm{P}-\mathrm{C}}=2.5 \mathrm{~Hz}\right)$, $72.9\left(\mathrm{~d},{ }^{2} J_{\mathrm{P}-\mathrm{C}}=11.5 \mathrm{~Hz}\right), 128.6\left(\mathrm{~d},{ }^{1} J_{\mathrm{P}-\mathrm{C}}=71.8 \mathrm{~Hz}\right), 128.6\left(\mathrm{~d},{ }^{2} J_{\mathrm{P}-\mathrm{C}}=12.3 \mathrm{~Hz}\right), 130.0(\mathrm{~d}$, $\left.{ }^{3} J_{\mathrm{P}-\mathrm{C}}=11.5 \mathrm{~Hz}\right), 131.5\left(\mathrm{~d},{ }^{4} J_{\mathrm{P}-\mathrm{C}}=3.3 \mathrm{~Hz}\right), 136.8\left(\mathrm{~d},{ }^{1} J_{\mathrm{P}-\mathrm{C}}=75.9 \mathrm{~Hz}\right), 157.3\left(\mathrm{~d},{ }^{2} J_{\mathrm{P}-\mathrm{C}}=23.0\right.$ 
$\mathrm{Hz}) ;{ }^{31} \mathrm{P}\left\{{ }^{1} \mathrm{H}\right\}$ NMR $\delta+68.9$; IR (KBr) $v_{\max } 3390(\mathrm{OH}) \mathrm{cm}^{-1}$; MS (MALDI-TOF) $\mathrm{m} / \mathrm{z} 314$ $\left([\mathrm{M}-2(\mathrm{OH})]^{+}\right)$. HRMS: Calcd for $\mathrm{C}_{19} \mathrm{H}_{25} \mathrm{O}_{2} \mathrm{PS}\left(\mathrm{M}^{+}\right), 348.1313$; Found, 348.1317.

Phosphole 9: A mixture of $4 \mathrm{~g}$ (53 mg, $0.15 \mathrm{mmol})$, elemental sulfur ( $24 \mathrm{mg}, 0.75 \mathrm{mmol})$, and $\mathrm{CH}_{2} \mathrm{Cl}_{2}(2 \mathrm{~mL})$ was stirred for 5 days at room temperature. The solvent was then evaporated, and the crude product was purified on a silica gel column using $\mathrm{CH}_{2} \mathrm{Cl}_{2}$ as an eluent $\left(R_{\mathrm{f}}=0.2\right)$ to give $\mathbf{9}(20 \mathrm{mg}, 35 \%)$ as a yellow solid. Mp $111-112{ }^{\circ} \mathrm{C} ;{ }^{1} \mathrm{H}$ NMR $\delta 1.16$ (t, 3H, J= 7.1 Hz), 2.35 (m, 2H), $2.91(\mathrm{~m}, 2 \mathrm{H}), 3.10(\mathrm{~m}, 2 \mathrm{H}), 4.14(\mathrm{~m}, 2 \mathrm{H}), 7.00(\mathrm{~m}, 1 \mathrm{H})$, $7.38(\mathrm{~m}, 1 \mathrm{H}), 7.42(\mathrm{~m}, 1 \mathrm{H}), 7.42(\mathrm{~m}, 2 \mathrm{H}), 7.50(\mathrm{~m}, 1 \mathrm{H}), 7.86(\mathrm{~m}, 2 \mathrm{H}) ;{ }^{13} \mathrm{C}\left\{{ }^{1} \mathrm{H}\right\} \mathrm{NMR} \delta$ 14.0, $27.0\left(\mathrm{~d},{ }^{4} J_{\mathrm{P}-\mathrm{C}}=1.7 \mathrm{~Hz}\right), 29.4\left(\mathrm{~d},{ }^{3} J_{\mathrm{P}-\mathrm{C}}=9.9 \mathrm{~Hz}\right), 30.9\left(\mathrm{~d},{ }^{3} J_{\mathrm{P}-\mathrm{C}}=9.1 \mathrm{~Hz}\right), 60.5,120.4$ $\left(\mathrm{d},{ }^{1} J_{\mathrm{P}-\mathrm{C}}=81.3 \mathrm{~Hz}\right), 128.0,128.4,128.6\left(\mathrm{~d},{ }^{2} J_{\mathrm{P}-\mathrm{C}}=13.2 \mathrm{~Hz}\right), 129.1\left(\mathrm{~d},{ }^{3} J_{\mathrm{P}-\mathrm{C}}=4.1 \mathrm{~Hz}\right)$, $129.2\left(\mathrm{~d},{ }^{1} J_{\mathrm{P}-\mathrm{C}}=82.5 \mathrm{~Hz}\right), 130.5,130.5\left(\mathrm{~d},{ }^{3} J_{\mathrm{P}-\mathrm{C}}=12.4 \mathrm{~Hz}\right), 132.1\left(\mathrm{~d},{ }^{4} J_{\mathrm{P}-\mathrm{C}}=2.8 \mathrm{~Hz}\right)$, $135.1\left(\mathrm{~d},{ }^{2} J_{\mathrm{P}-\mathrm{C}}=18.2 \mathrm{~Hz}\right), 149.7\left(\mathrm{~d},{ }^{2} J_{\mathrm{P}-\mathrm{C}}=19.8 \mathrm{~Hz}\right), 162.0\left(\mathrm{~d},{ }^{2} J_{\mathrm{P}-\mathrm{C}}=17.4 \mathrm{~Hz}\right), 173.6(\mathrm{~d}$, $\left.{ }^{2} J_{\mathrm{P}-\mathrm{C}}=19.8 \mathrm{~Hz}\right) ;{ }^{31} \mathrm{P}\left\{{ }^{1} \mathrm{H}\right\} \mathrm{NMR} \delta+66.2 ; \mathrm{IR} v_{\max } 1692(\mathrm{C}=\mathrm{O}) \mathrm{cm}^{-1} ;$ MS (MALDI-TOF) m/z $387\left(\mathrm{M}^{+}\right)$. HRMS: Calcd for $\mathrm{C}_{20} \mathrm{H}_{19} \mathrm{O}_{2} \mathrm{PS}_{2}, 386.0564\left(\mathrm{M}^{+}\right)$; Found, 386.0566.

Phosphole 10: To a solution of $\mathbf{4 g}$ (200 $\mathrm{mg}, 0.56 \mathrm{mmol})$ in $10 \mathrm{~mL}$ of hexane was added a hexane solution of DIBAL $(1.0 \mathrm{M}, 1.12 \mathrm{~mL}, 1.12 \mathrm{mmol})$ at $-78^{\circ} \mathrm{C}$. After stirring for $1 \mathrm{~h}$ at $-50{ }^{\circ} \mathrm{C}$, elemental sulfur $(27 \mathrm{mg}, 0.85 \mathrm{mmol})$ was added, and the resulting mixture was allowed to warm slowly to room temperature. A saturated $\mathrm{NH}_{4} \mathrm{Cl}$ solution $(5 \mathrm{~mL})$ was then added, and the mixture was filtered through a Celite bed. The organic phase was separated from the filtrate, and the aqueous phase was extracted with $\mathrm{Et}_{2} \mathrm{O}(10 \mathrm{~mL} \times 2)$. The combined organic extracts were treated with brine $(20 \mathrm{~mL})$, dried over $\mathrm{MgSO}_{4}$. and evaporated to give a crude product, which was purified on a silica gel column using hexane/AcOEt $(2: 1)$ as eluents $\left(R_{\mathrm{f}}=0.3\right)$ to afford $10(160 \mathrm{mg}, 83 \%)$ as a yellow solid. 
Mp 170-171 ${ }^{\circ} \mathrm{C} ;{ }^{1} \mathrm{H}$ NMR $\delta 2.09$ (br, 1H), 2.31 (m, 2H), 2.72 (m, 2H), 2.87 (m, 2H), 4.48 (m, 2H), $6.95(\mathrm{~m}, 1 \mathrm{H}), 7.25(\mathrm{~m}, 1 \mathrm{H}), 7.29(\mathrm{~m}, 1 \mathrm{H}), 7.43(\mathrm{~m}, 2 \mathrm{H}), 7.50(\mathrm{~m}, 1 \mathrm{H}), 7.88(\mathrm{~m}$, $2 \mathrm{H}) ;{ }^{13} \mathrm{C}\left\{{ }^{1} \mathrm{H}\right\}$ NMR $\delta 27.2\left(\mathrm{~d},{ }^{4} J_{\mathrm{P}-\mathrm{C}}=1.7 \mathrm{~Hz}\right), 27.5\left(\mathrm{~d},{ }^{3} J_{\mathrm{P}-\mathrm{C}}=11.7 \mathrm{~Hz}\right), 29.7\left(\mathrm{~d},{ }^{3} J_{\mathrm{P}-\mathrm{C}}=\right.$ $11.1 \mathrm{~Hz}), 57.7\left(\mathrm{~d},{ }^{2} J_{\mathrm{P}-\mathrm{C}}=15.0 \mathrm{~Hz}\right), 125.0\left(\mathrm{~d},{ }^{1} J_{\mathrm{P}-\mathrm{C}}=81.3 \mathrm{~Hz}\right), 126.4,126.7\left(\mathrm{~d},{ }^{3} J_{\mathrm{P}-\mathrm{C}}=4.5\right.$ $\mathrm{Hz}), 127.6,127.8,128.4\left(\mathrm{~d},{ }^{1} J_{\mathrm{P}-\mathrm{C}}=71.4 \mathrm{~Hz}\right), 128.9\left(\mathrm{~d},{ }^{2} J_{\mathrm{P}-\mathrm{C}}=12.8 \mathrm{~Hz}\right), 130.4\left(\mathrm{~d},{ }^{3} J_{\mathrm{P}-\mathrm{C}}=\right.$ $11.7 \mathrm{~Hz}), 132.1\left(\mathrm{~d},{ }^{4} J_{\mathrm{P}-\mathrm{C}}=2.8 \mathrm{~Hz}\right), 135.2\left(\mathrm{~d},{ }^{2} J_{\mathrm{P}-\mathrm{C}}=19.5 \mathrm{~Hz}\right), 151.9\left(\mathrm{~d},{ }^{2} J_{\mathrm{P}-\mathrm{C}}=22.8 \mathrm{~Hz}\right)$, $158.2\left(\mathrm{~d},{ }^{2} J_{\mathrm{P}-\mathrm{C}}=21.7 \mathrm{~Hz}\right) ;{ }^{31} \mathrm{P}\left\{{ }^{1} \mathrm{H}\right\} \mathrm{NMR} \delta+68.0 ; \mathrm{IR}(\mathrm{KBr}) v_{\max } 3475(\mathrm{OH}) \mathrm{cm}^{-1} ; \mathrm{MS}$ (MALDI-TOF) $\mathrm{m} / \mathrm{z} 344\left(\mathrm{M}^{+}\right)$. Anal. Calcd for $\mathrm{C}_{18} \mathrm{H}_{17} \mathrm{OPS}_{2}$ : C, 62.77; H, 4.97; P, 8.99. Found: C, 62.58; H, 5.02; P, 8.73. 


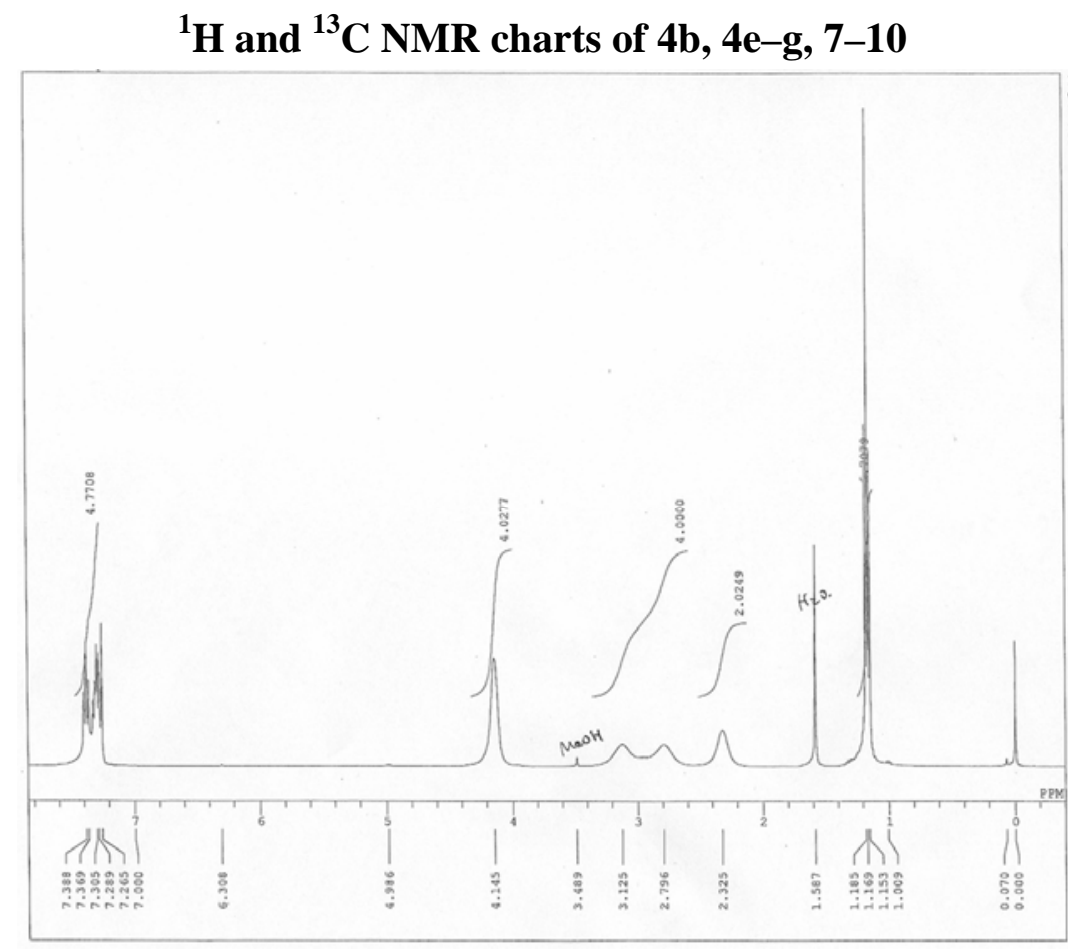

Figure S1. ${ }^{1} \mathrm{H}$ NMR chart of $4 \mathrm{~b}$.

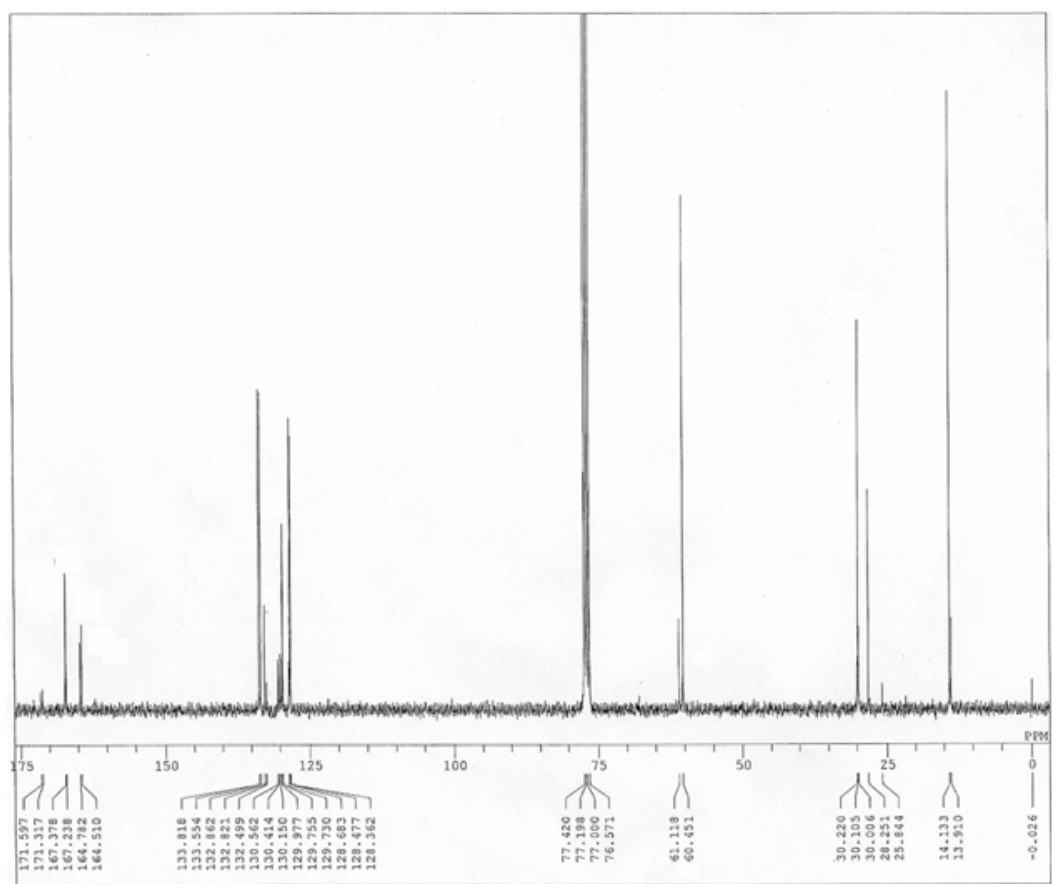

SI- $\quad 10$ 
Figure S2. ${ }^{13} \mathrm{C}$ NMR chart of $4 \mathrm{~b}$.

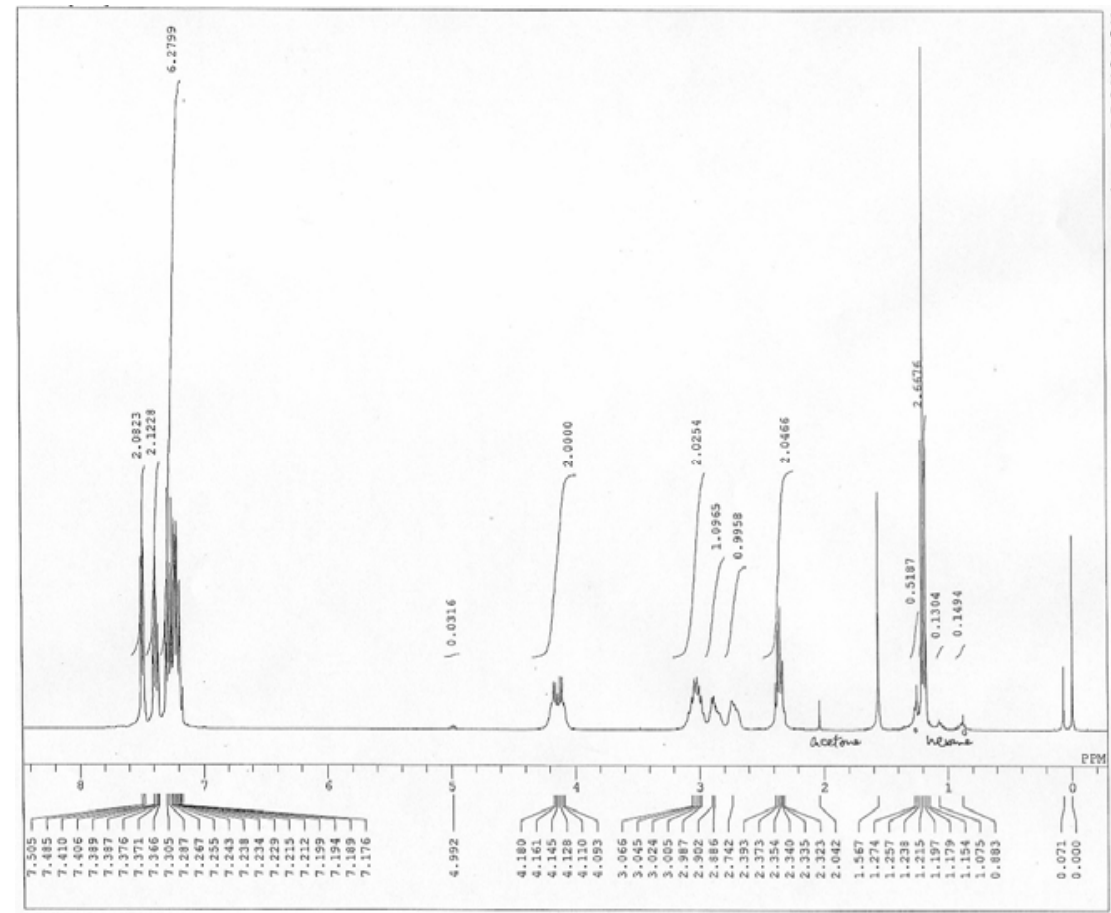

Figure S3. ${ }^{1} \mathrm{H}$ NMR chart of $4 \mathrm{e}$.

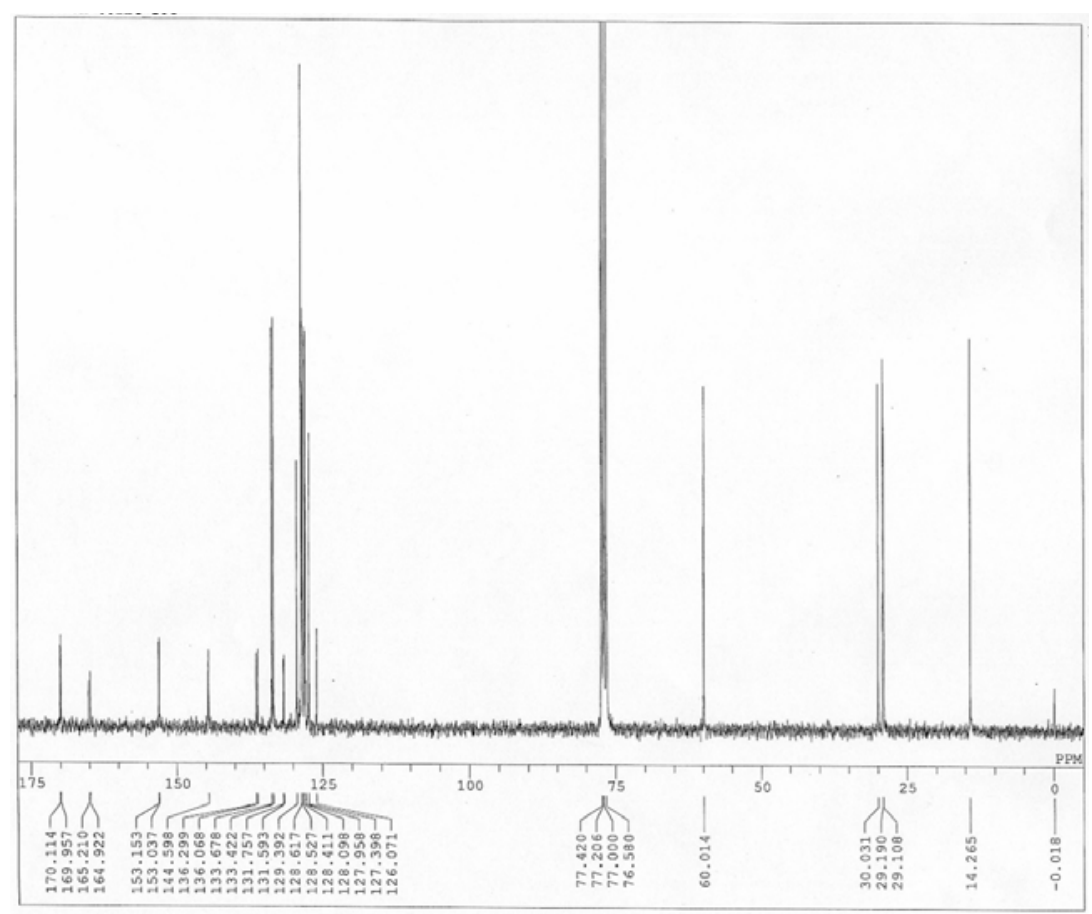

Figure S4. ${ }^{13} \mathrm{C}$ NMR chart of $4 \mathrm{e}$.

SI- 


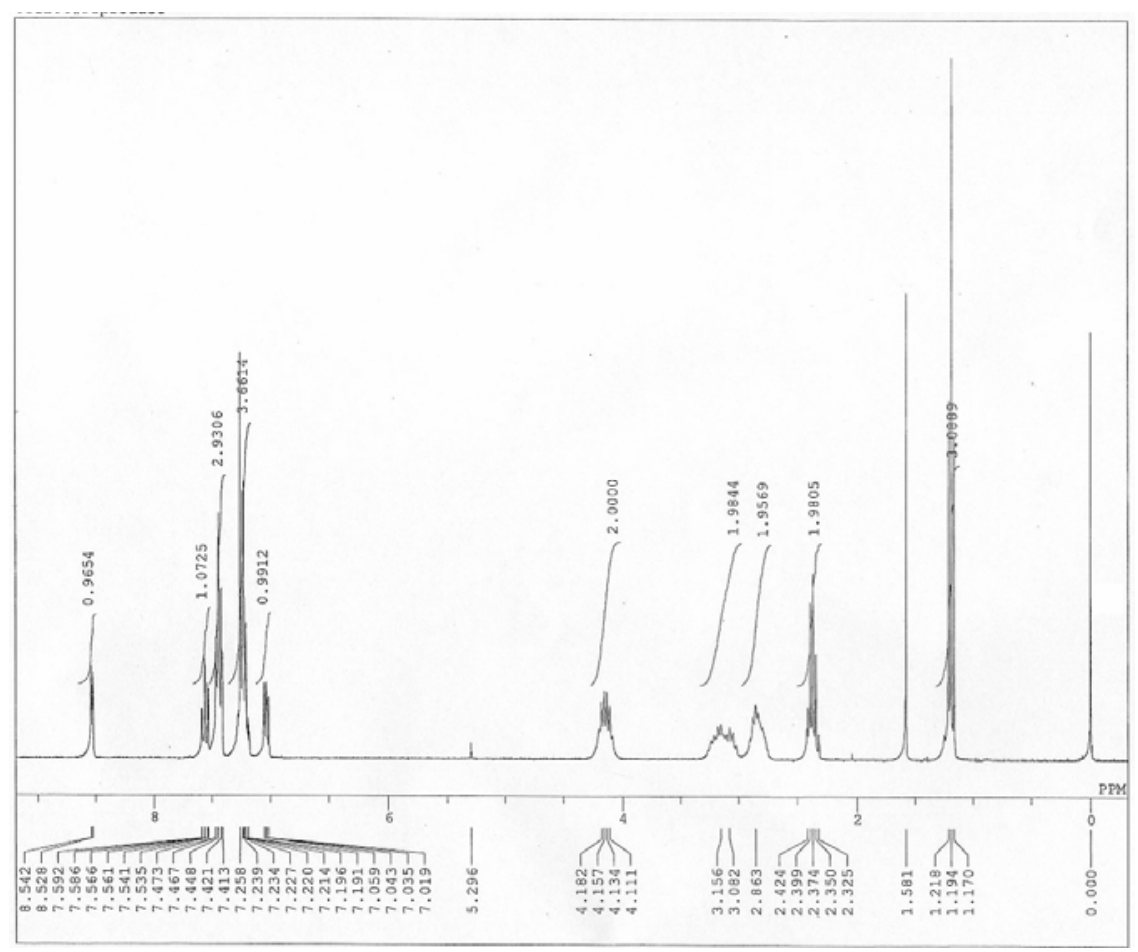

Figure S5. ${ }^{1} \mathrm{H}$ NMR chart of $4 \mathrm{f}$.

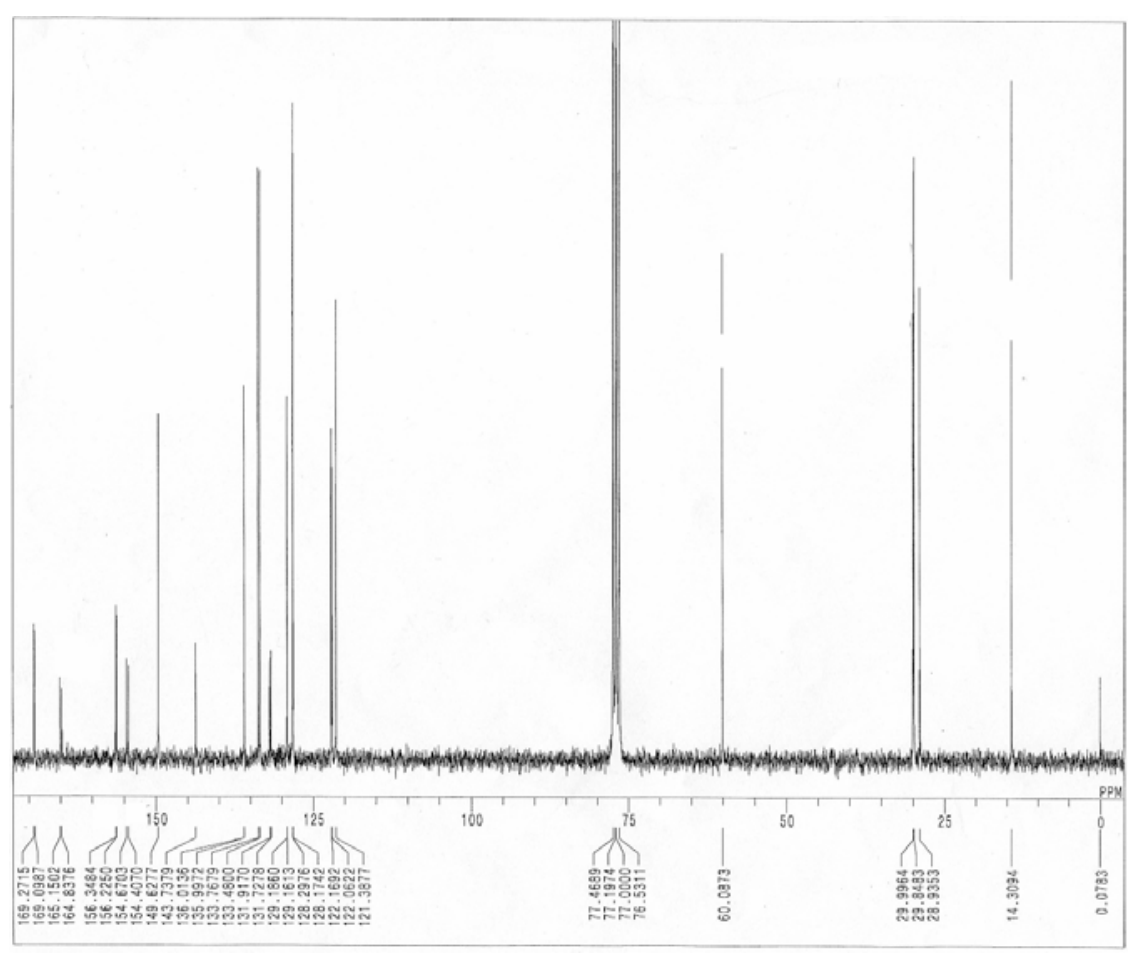

Figure S6. ${ }^{13} \mathrm{C}$ NMR chart of $4 \mathrm{f}$.

SI- $\quad 12$ 


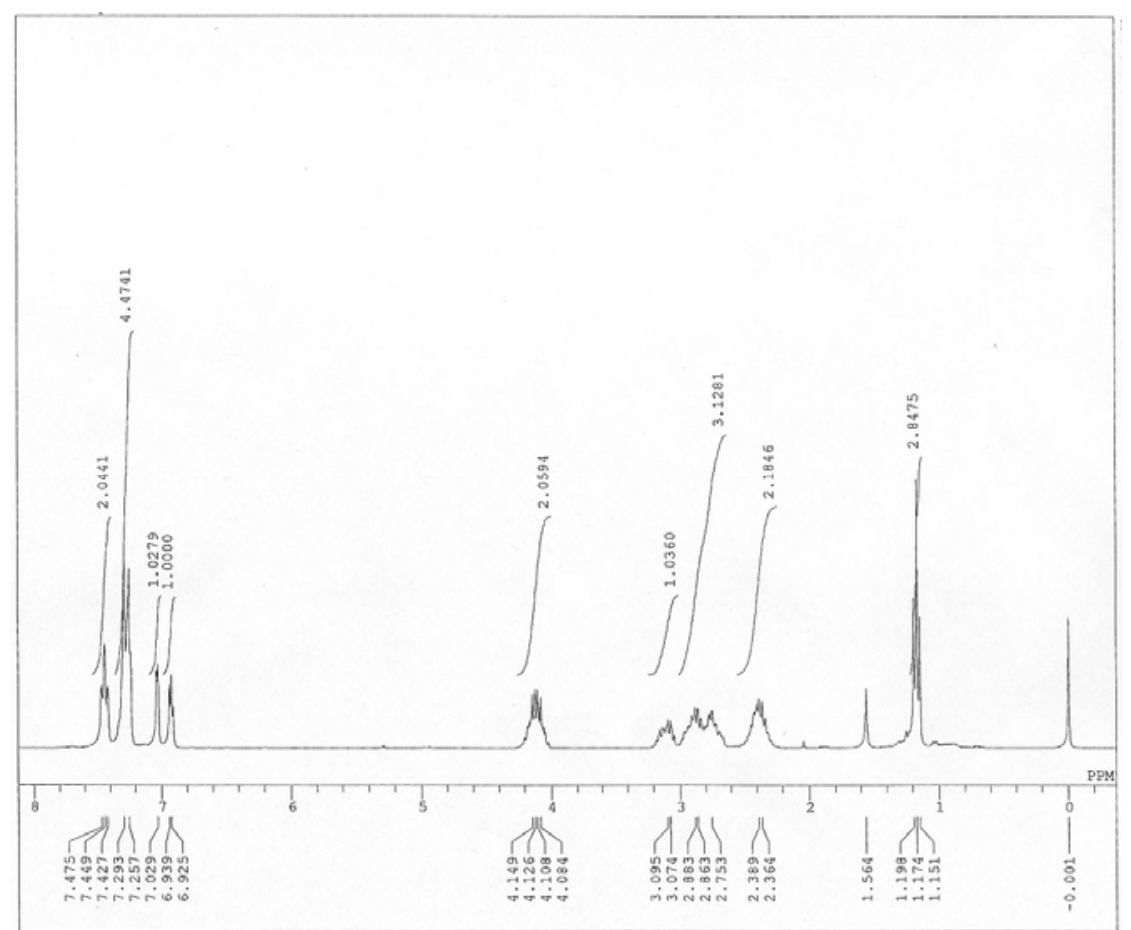

Figure S7. ${ }^{1} \mathrm{H}$ NMR chart of $\mathbf{4 g}$.

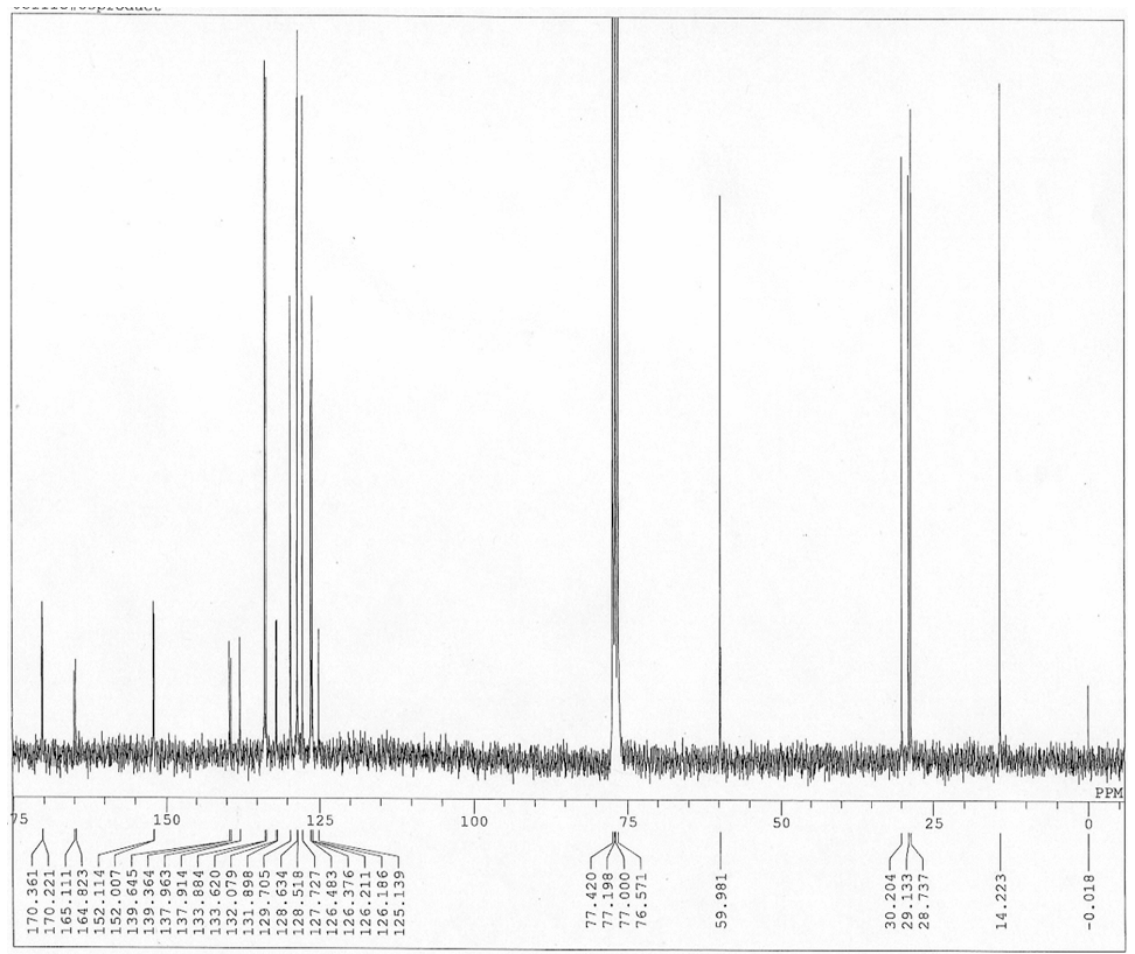

Figure S8. ${ }^{13} \mathrm{C}$ NMR chart of $\mathbf{4 g}$.

SI- 


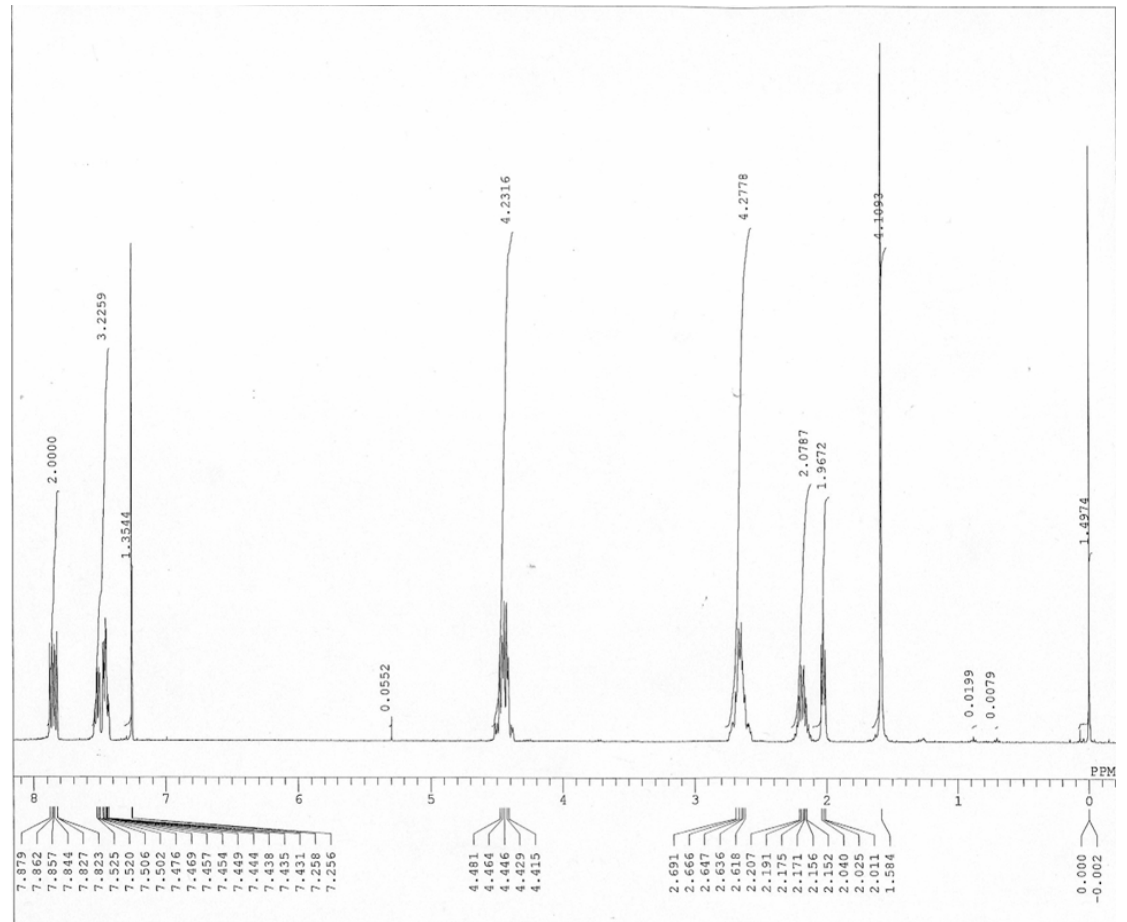

Figure S9. ${ }^{1} \mathbf{H}$ NMR chart of 7.

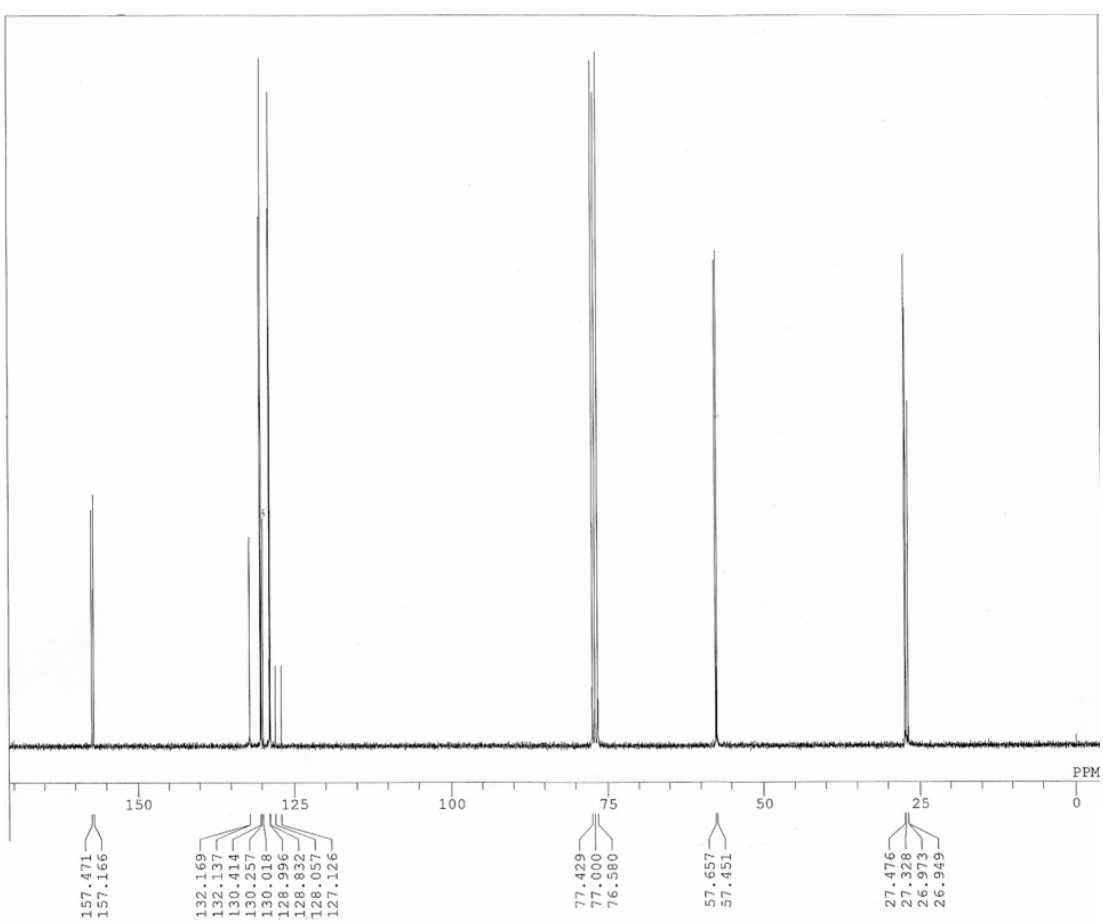

Figure S10. ${ }^{13} \mathrm{C}$ NMR chart of 7.

SI- $\quad 14$ 


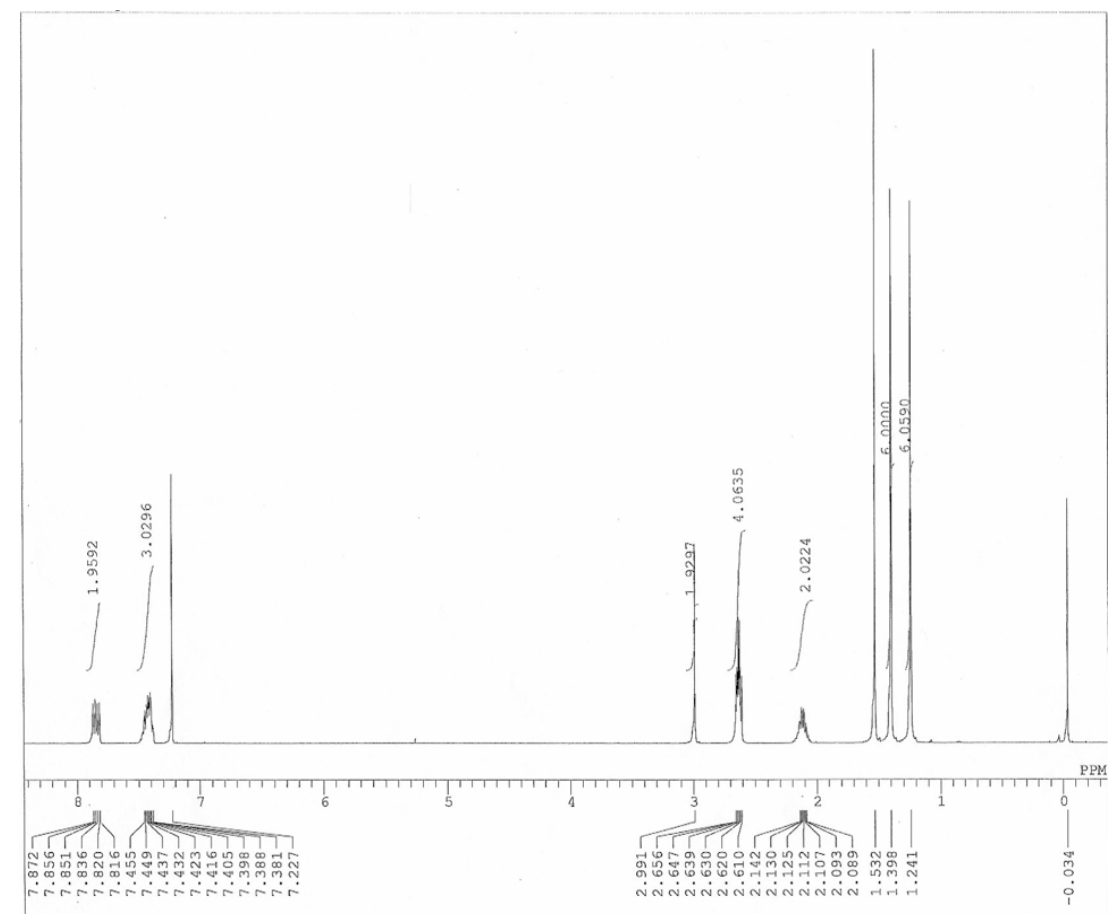

Figure S11. ${ }^{1} \mathrm{H}$ NMR chart of 8.

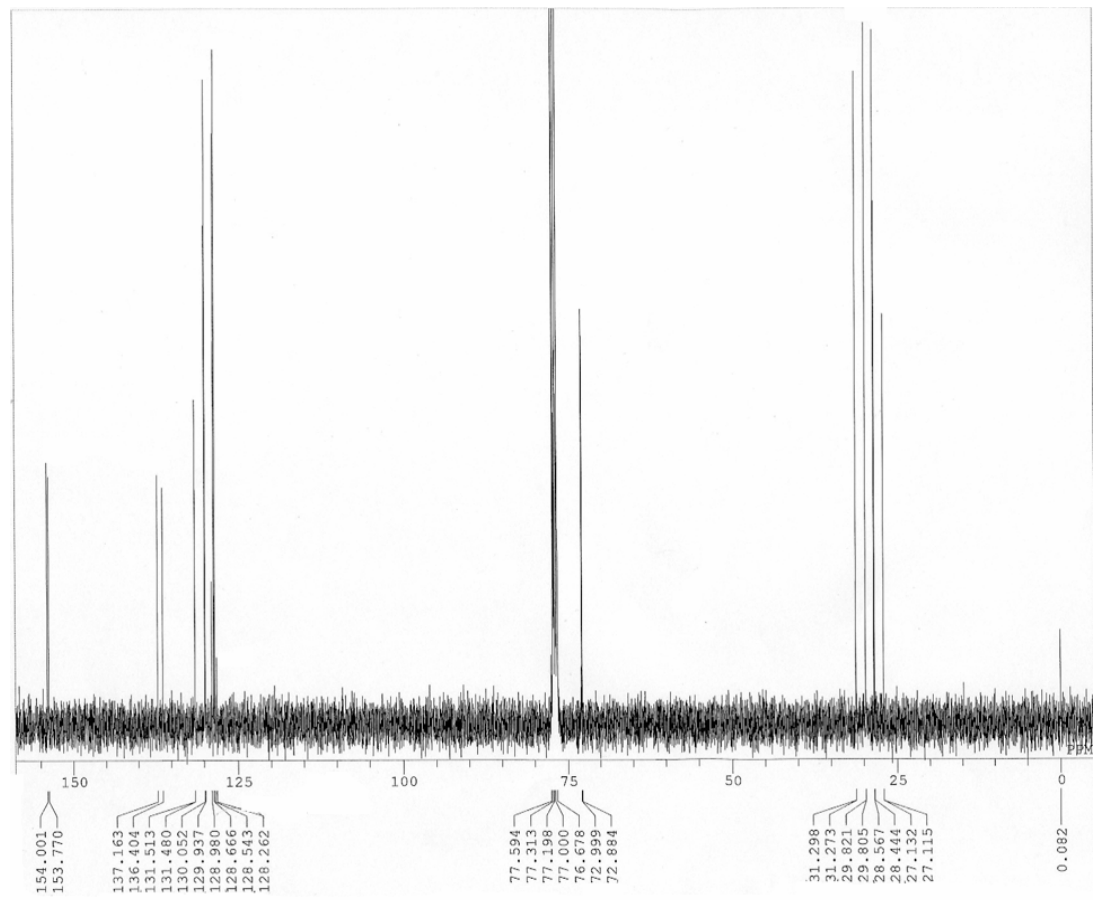

Figure S12. ${ }^{13} \mathrm{C}$ NMR chart of 8.

SI- 


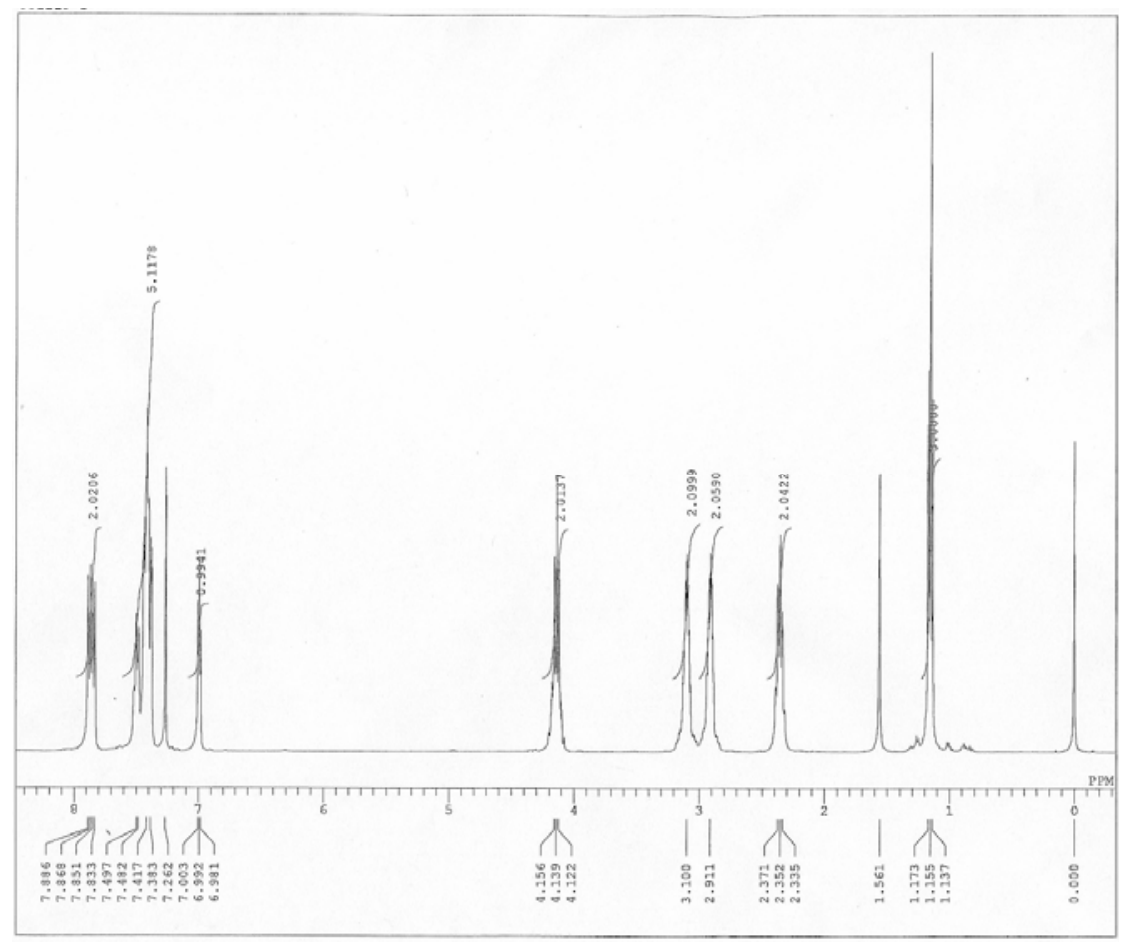

Figure S13. ${ }^{1} \mathrm{H}$ NMR chart of 9 .

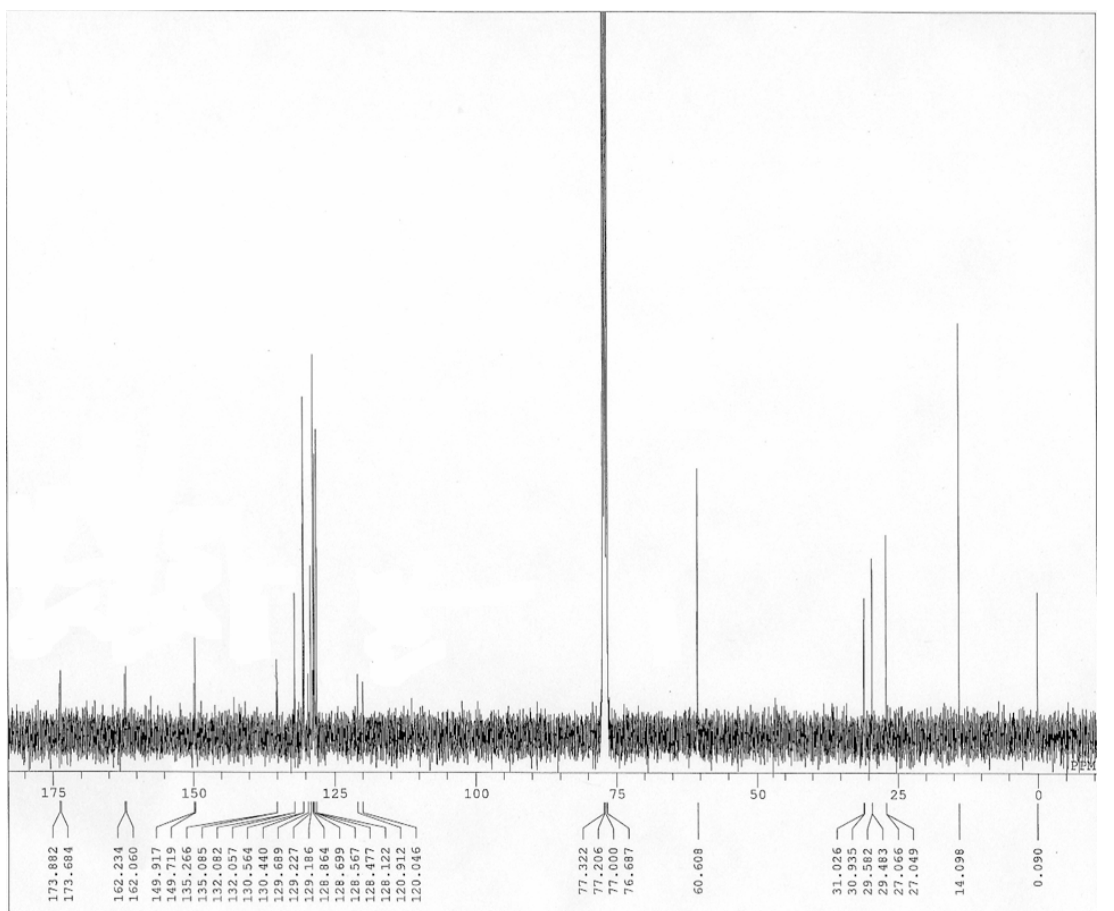

Figure S14. ${ }^{13} \mathrm{C}$ NMR chart of 9 .

SI- 


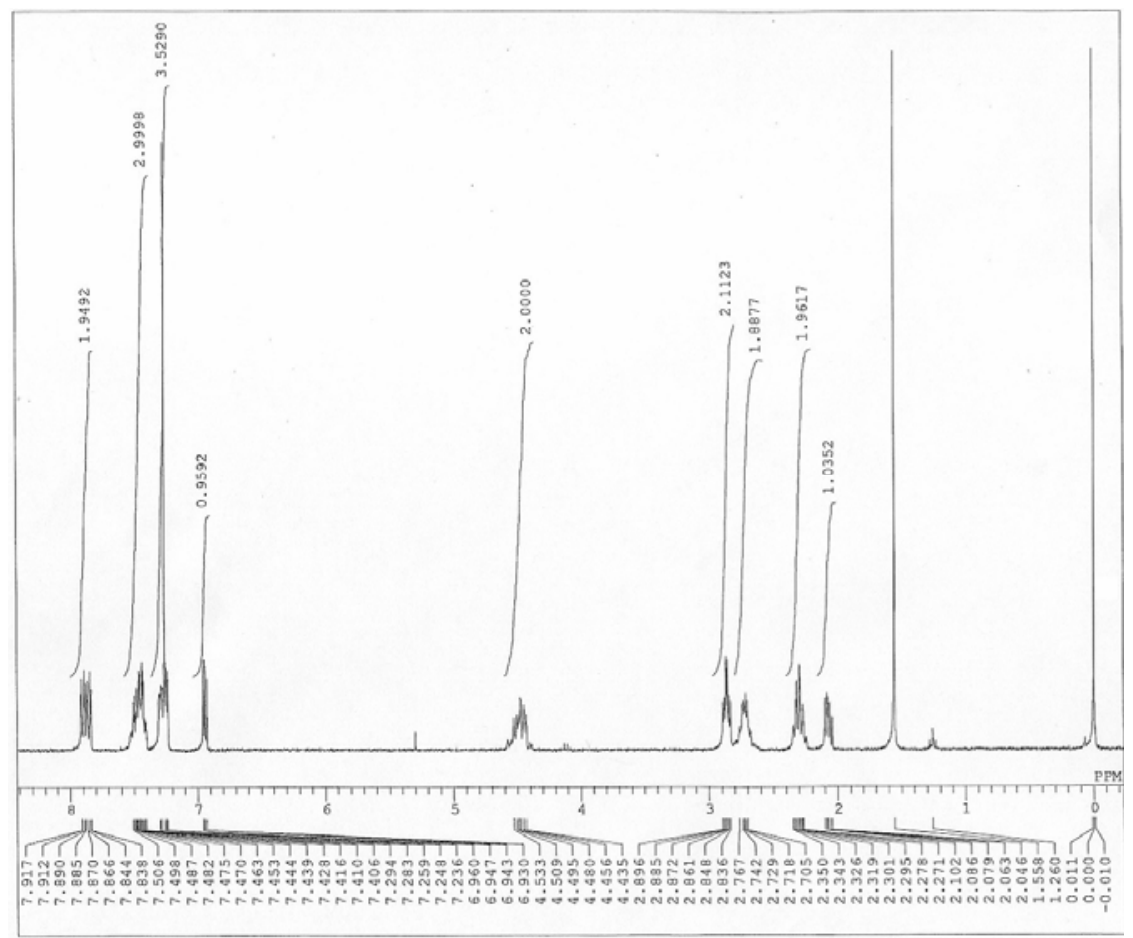

Figure S15. ${ }^{1} \mathrm{H}$ NMR chart of 10.

SI- $\quad 17$ 